\title{
DEPRESSÃO NA ADOLESCÊNCIA E COMPORTAMENTO SUICIDA: UMA REVISÃO INTEGRATIVA
}

Aline Sharlon Maciel Batista Ramos ${ }^{1}$; Suziane Mendonça Mesquita ${ }^{2}$; Débora Luana Ribeiro Pessoa ${ }^{3}$; Rafael Mondego Fontenele ${ }^{4}$; Isabela Bastos Jácome de Sousa ${ }^{5}$.

1 Enfermeira. Doutoranda em Ciências (UERJ). Professora Assistente da

Universidade CEUMA. E-mail: alinesharlon@gmail.com

2 Discente do 5ำ ano do Curso de Enfermagem da Universidade CEUMA.

3 Farmacêutica, Doutorado em Biotecnologia. Professora Adjunta da Universidade

Federal do Maranhão (UFMA).

4 Enfermeiro. Especialista em Saúde da Família. Mestrando em Gestão de

Programas e Serviços de Saúde (Universidade CEUMA).

5 Enfermeira. Mestre em Saúde da Família (UNINOVAFAPI). Professora Assistente da Universidade CEUMA

Recebido em: 06/04/2018 - Aprovado em: 10/06/2018 - Publicado em: 20/06/2018 DOI: 10.18677/EnciBio_2018A123

\begin{abstract}
RESUMO
A depressão na adolescência é considerada um problema de saúde pública, devido a sua elevada prevalência e a tendência de o transtorno mental apresentar longa duração e recorrência. Vale ressaltar que diversos fatores podem tornar o adolescente vulnerável, levando ao desenvolvimento da depressão e como conseqüência mais grave o suicídio. Foi realizada uma revisão Integrativa de Literatura com busca na base de dados: Scientific Eletronic Library Online (SciELO) através dos descritores: depressão e adolescentes. Foram encontrados 101 artigos e 07 se enquadraram nos critérios de inclusão. Buscou-se responder a seguinte questão norteadora: Quais são as evidências cientificas nacionais publicados nos últimos dez anos que contribuem para a compreensão da sintomatologia depressiva manifestada na adolescência e sua associação com o comportamento suicida? Os artigos evidenciaram que os adolescentes estão mais suscetíveis a manifestar a sintomatologia depressiva, são aqueles que estão expostos a diversos tipos de violências, eventos estressores ao longo da vida, a questões ligadas a classe social baixa,vivenciar separação dos pais e baixo rendimento escolar, tornando o jovem vulnerável a comportamentos suicida.Os estudos confirmaram uma elevada prevalência no quadro depressivo vivenciados por essa população, com maior freqüência no sexo feminino. Diante do exposto, sugere-se que no ambiente escolar sejam articuladas estratégias de enfrentamento junto aos educadores e profissionais de saúde, para que façam abordagem sobre essa temática, com a finalidade de identificação precoce dos sintomas e prevenção de outras doenças psíquicas.
\end{abstract}

PALAVRAS-CHAVE: Adolescentes. Depressão. Suicídio. 


\title{
DEPRESSION IN ADOLESCENCE AND SUICIDE BEHAVIOR: AN INTEGRATING REVIEW
}

\begin{abstract}
Adolescence depression is considered a public health problem due to its high prevalence and the tendency for mental disorder to present long duration and recurrence. It is noteworthy that several factors can make adolescents vulnerable, leading to the development of depression and as a consequence more serious suicide. An Integrative Review of Literature with search in the database was conducted: Scientific Electronic Library Online (SciELO) through the descriptors: depression and adolescents. 101 articles were found and 07 were included in the inclusion criteria. We sought to answer the following guiding question: What are the national scientific evidences published in the last ten years that contribute to the understanding of the depressive symptomatology manifested in adolescence and its association with suicidal behavior? The articles showed that adolescents are more susceptible to manifest depressive symptoms, are those who are exposed to different types of violence, stressful events throughout life, issues related to low social class, experiencing parental separation and low school performance, making the young person vulnerable to suicidal behavior. The studies confirmed a high prevalence in the depressive picture experienced by this population, more frequently in the female sex. In view of the above, it is suggested that in the school environment strategies of coping with the educators and health professionals should be articulated to address this issue, with the purpose of early identification of symptoms and prevention of other psychic diseases.
\end{abstract}

KEYWORDS: Adolescents. Depression. Suicide.

\section{INTRODUÇÃO}

De acordo com a Organização Mundial de Saúde (OMS) e a Organização Pan Americana de Saúde (OPAS), a depressão tem representado um crescente problema para a saúde pública mundial. Globalmente estima-se que 350 milhões de pessoas de todas as idades sofram com esse transtorno e que até 2020 seja a segunda causa de incapacidade mental em termos mundiais para a saúde pública (WHO, 2016).

O Instituto Nacional de Saúde Mental (NIMH) dos Estados Unidos, a partir da década de 1970, apontou a depressão infantil como de fato uma patologia, o que aumentou de forma significativa o número de estudos a seu respeito. Entretanto, a depressão em crianças e adolescentes passou a ser vista como uma preocupação entre os profissionais de saúde, sobretudo devido aos comprometimentos que acarreta no desenvolvimento social, emocional e cognitivo do indivíduo (GOMES, 2013).

No Brasil, segundo o relatório global da Organização Mundial de Saúde, as estatísticas evidenciam que a depressão atinge $5,8 \%$ de sua população. De acordo com o mesmo relatório, nas complicações mais graves, a depressão pode levar ao suicídio e cerca de 800 mil pessoas morrem a cada ano sendo a segunda principal causa de morte entre pessoas com idade de 15 a 29 anos, acometendo grande parte dos adolescentes (WHO, 2016).

A depressão pode ser caracterizada por transtorno de humor grave com prejuízo da função mental e com distorção da forma como a pessoa vivencia e 
entende a realidade. Ocorre em todas as faixas etárias, acometendo principalmente adolescentes e idosos (MIRANDA, 2013).

A adolescência é o período de transição para a idade adulta, repleta de alterações morfológicas e psicossociais. Cronologicamente esta fase corresponde ao período dos 10 aos 19 anos de idade (MIRANDA et al, 2014).

Diante deste cenário, alguns fatores de riscos são apresentados pela literatura, que podem potencializar ou são protetores para os sintomas depressivos, como a associação entre a presença de problemas psíquicos e agressividade, bullying escolar e a dependência ao acesso à internet com uso de meios eletrônicos, e os ataques cibernéticos que afetam a integridade social dos adolescentes (BOTINO et al., 2015).

Marques e Helliwell (2015) afirmam ainda que fatos traumáticos ocorridos na infância, como perdas de vínculos afetivos devido à morte, separação dos pais e abandono estão entre os fatores que predispõem a depressão, devendo-se considerar também o histórico familiar de depressão em um dos pais aumenta os riscos em ate três vezes, seguidos por estressores ambientais, como abuso físico e sexual.

Todavia, a depressão tem se tornado preocupante devido a sua prevalência elevada, longa duração e recorrência acarretando disfunções sociais e ocupacionais prolongadas, alto grau de morbidade e de atentados contra a própria vida, além disso, o desenvolvimento de transtornos mentais pode produzir diversos danos psicossociais para o adolescente (KOLVALSKI, 2015).

Considera-se que os sintomas depressivos apresentam natureza duradoura e pervasiva, afetando múltiplas funções e causando significativos danos psicossociais, irritabilidade, desmotivação, desinteresse, humor deprimido e instável o que pode apresentar crises de explosão de raiva. (BIAZUS et al.,2016).

De um modo geral, a depressão pode aparecer como um sintoma secundário de determinada doença, ou mesmo coexistir junto com outros estados emocionais como causa de eventos traumatizantes, ou ainda como agravo isolado, e pode ser associado ao suicídio como consequência mais grave (KOLVALSKI, 2015). Sendo assim estudos relacionados ao suicídio mencionam a depressão como um dos principais fatores de riscos em todas as faixas etárias tornando-se relevante, pois, esse transtorno desempenha um importante papel diante dos atos suicidas (BRAGA, 2013; DELL'AGLIO et al., 2013).

O objetivo desta pesquisa foi identificar o comportamento suicida nos estudos já publicados.

\section{MATERIAL E MÉTODOS}

Trata-se de revisão integrativa da literatura realizada a partir da definição da questão de pesquisa, buscas em bases de dados, categorização e avaliação dos estudos inclusos na pesquisa, interpretação e apresentação dos resultados. A revisão integrativa possui como finalidade uma abordagem metodológica que permite a inclusão de estudos experimentais e não experimentais para a compreensão do fenômeno analisado (SOUZA et. al., 2010).

A questão norteadora desta revisão foi: quais são as evidências científicas nacionais publicadas nos últimos 10 anos que contribuem para a compreensão da sintomatologia depressiva manifestada na adolescência e sua correlação com comportamento suicida? 
Para seleção dos artigos realizaram-se buscas na base de dado SCIELO. A estratégia de busca foi realizada pelas palavras-chave dos Descritores em Ciências da Saúde (DECS): depressão e adolescentes.

Definiu-se como critérios de inclusão artigos nacionais publicados em português entre 2006 a 2016, com os textos completos disponíveis nas bases de dados indexadas e que retratam a questão de adolescentes com história de depressão. Como critérios de exclusão foram eleitos: publicações anteriores ao ano 2006, ausência de acesso gratuito ao texto completo on-line, sem restrição de etnicidade, divulgados na língua portuguesa e artigos duplicados. A busca em base de dados foi realizada no mês de agosto de 2017. A amostra final desta revisão foi constituída por sete artigos científicos, selecionados pelos critérios de inclusão previamente estabelecidos.

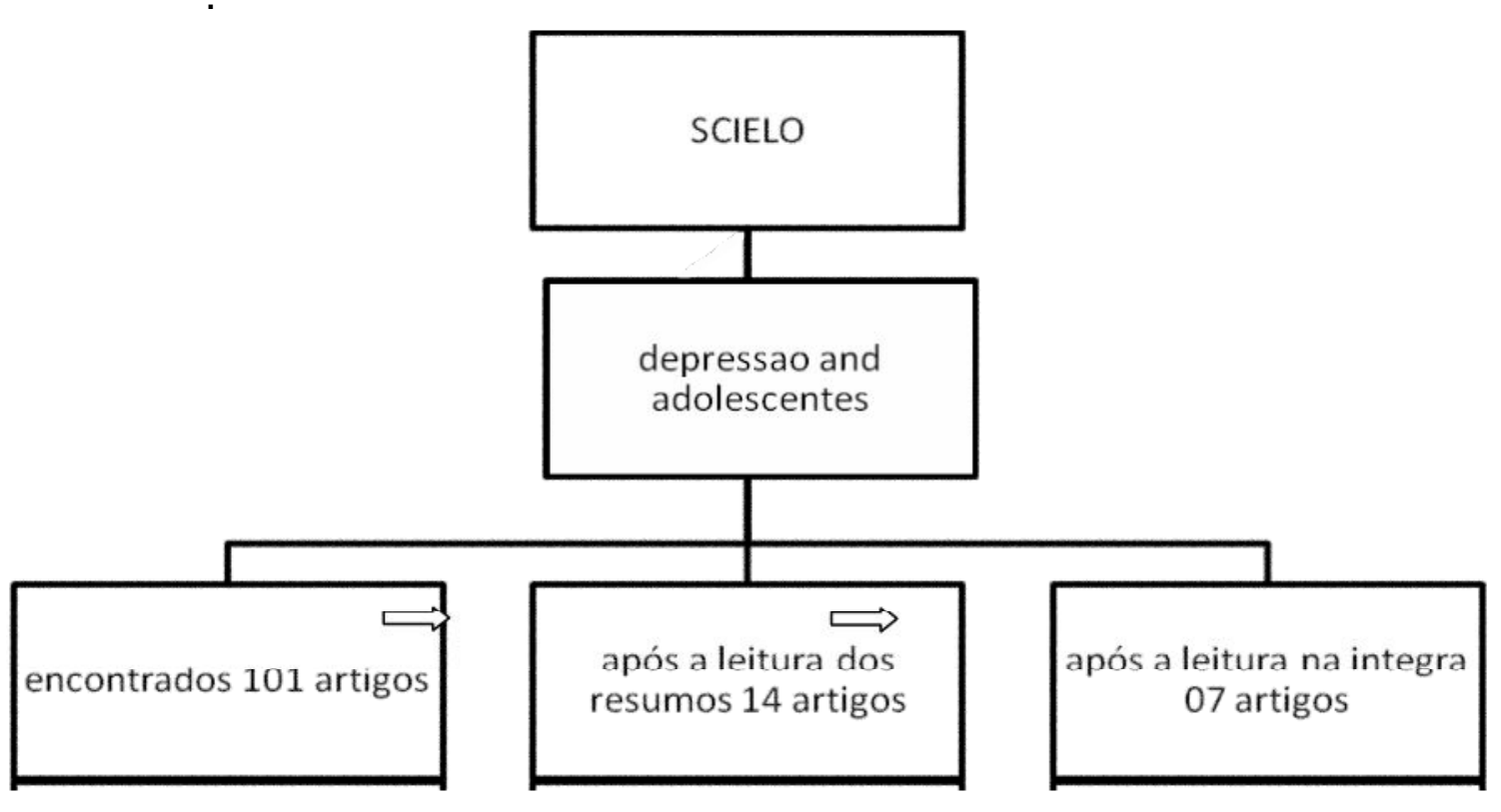

Figura 01- Descrição da seleção dos artigos utilizados no estudo.

\section{RESULTADOS E DISCUSSÃO}

Por meio de busca eletrônica foram identificados 101 estudos, dos quais 94 artigos foram excluídos após a leitura de títulos e resumos. Configurou-se a amostra de estudo com um total de sete artigos encontrados na base de dados SCIELO. 
Quadro 01: Distribuição dos artigos selecionados: Base de dados, autores dos periódicos, ano de publicação e título.

\begin{tabular}{|c|c|c|c|c|}
\hline BASE & AUTOR & PERIODICO & ANO & TITULO \\
\hline SCIELO & $\begin{array}{l}\text { Joana D'Arc } \\
\text { Vila Nova } \\
\text { Jatobá; } \\
\text { Othon } \\
\text { Bastos. }\end{array}$ & $\begin{array}{l}\text { Jornal } \\
\text { brasileiro de } \\
\text { Psiquiatria }\end{array}$ & 2007 & $\begin{array}{l}\text { Depressão e ansiedade em } \\
\text { adolescentes de escolas } \\
\text { públicas e privadas, (A1). }\end{array}$ \\
\hline SCIELO & $\begin{array}{l}\text { Joviana } \\
\text { Q.Avanci; } \\
\text { Simone } \\
\text { G.Assis et } \\
\text { al. }\end{array}$ & $\begin{array}{l}\text { Cadernos de } \\
\text { Saúde Pública }\end{array}$ & 2008 & $\begin{array}{l}\text { Sintomas depressivos na } \\
\text { adolescência: estudo sobre os } \\
\text { fatores Psicossociais em } \\
\text { amostra de um município do Rio } \\
\text { de Janeiro, Brasil (A2). }\end{array}$ \\
\hline SCIELO & $\begin{array}{l}\text { Luciano D. } \\
\text { M. Souza; } \\
\text { Rodrigo S. } \\
\text { Silva et al. }\end{array}$ & $\begin{array}{ll}\text { Jornal } & \mathrm{da} \\
\text { Psiquiatria } & \end{array}$ & 2008 & $\begin{array}{l}\text { Sintomatologia depressiva em } \\
\text { adolescentes iniciais: estudo de } \\
\text { base populacional, (A3). }\end{array}$ \\
\hline SCIELO & $\begin{array}{ll}\text { Thais } & \text { A. } \\
\text { Aragão; } & \\
\text { Maria P. L. } \\
\text { Coutinho et } \\
\text { al. }\end{array}$ & $\begin{array}{l}\text { Ciência } \quad \text { e } \\
\text { Saúde } \\
\text { Coletiva }\end{array}$ & 2009 & $\begin{array}{l}\text { Uma perspectiva psicossocial } \\
\text { da sintomatologia depressiva na } \\
\text { adolescência, (A4). }\end{array}$ \\
\hline SCIELO & $\begin{array}{l}\text { Karla C. S. } \\
\text { Ribeiro; } \\
\text { Maria P. L. } \\
\text { Coutinho et } \\
\text { al. }\end{array}$ & $\begin{array}{l}\text { Psicologia } \\
\text { Ciência } \\
\text { Profissão }\end{array}$ & 2010 & $\begin{array}{l}\text { Representação social da } \\
\text { depressão em uma instituição } \\
\text { de ensino da rede pública, (A5). }\end{array}$ \\
\hline SCIELO & $\begin{array}{ll}\text { Natalia } & \text { F. } \\
\text { Damião; } & \\
\text { Maria P. } & \text { L. } \\
\text { Coutinho } & \text { et } \\
\text { al. } & \\
\end{array}$ & $\begin{array}{l}\text { Psicologia \& } \\
\text { Sociedade }\end{array}$ & 2011 & $\begin{array}{l}\text { Representações sociais da } \\
\text { depressão no ensino médio um } \\
\text { estudo sobre duas capitais, } \\
\text { (A6). }\end{array}$ \\
\hline SCIELO & $\begin{array}{ll}\text { Diego } & \text { G. } \\
\text { Barbosa; } & \\
\text { Rubian } & \text { D. } \\
\begin{array}{l}\text { Andrade, et } \\
\text { al. }\end{array} & \end{array}$ & $\begin{array}{l}\text { Caderneta de } \\
\text { Saúde Publica }\end{array}$ & 2016 & $\begin{array}{ll}\text { Sintomas depressivos em } \\
\text { adolescentes em situação de } \\
\text { vulnerabilidade social, (A7). }\end{array}$ \\
\hline
\end{tabular}

Todos os estudos selecionados foram realizados com adolescentes, em fase escolar e de escolas públicas e privadas do Brasil, com média de idade entre 14 e 19 anos. Apenas um estudo do tipo quanti-qualitativo estendeu a faixa etária até 25 anos de idade, este estudo utilizou as Representações Sociais (RS) e o Teste de Associação Livre de Palavras (TALP), e para aprofundar as análises estatísticas acerca dos dados coletados, utilizou o teste T Student com o objetivo de identificar os fatores psicossociais que interferem na etiologia da depressão e apreender as representações sociais da depressão no coletivo de adolescentes inseridos no contexto do ensino médio da rede pública e privada das cidades de Teresina-PI e Natal-RN.

Os estudos foram classificados de acordo com o ano de publicação na ordem crescente. 
Quadro 02- apresentada a distribuição dos estudos de acordo com amostra e principais resultados.

\begin{tabular}{|c|c|c|}
\hline ARTIGO & $\begin{array}{l}\text { DELINEAMENTO DA } \\
\text { AMOSTRA }\end{array}$ & PRINCIPAIS RESULTADOS \\
\hline A1 & $\begin{array}{l}\text { Amostra realizada com } \\
242 \text { estudantes, na faixa } \\
\text { etária de } 14 \text { a } 16 \text { anos, } \\
\text { em escolas públicas e } \\
\text { privadas. }\end{array}$ & $\begin{array}{l}\text { Nesse estudo identificou-se que } 68,6 \% \\
\text { dos adolescentes apresentaram humor } \\
\text { deprimido, } 61,6 \% \text { sentimentos de culpas } \\
\text { expressos por auto-recriminação e } 50,8 \% \\
\text { manifestaram agitação caracterizada por } \\
\text { inquietude e desassossego, ainda } 83 \\
\text { alunos }(34,3 \%) \text {, referiram ideação suicida } \\
\text { ou mesmo tentativa de suicídio. }\end{array}$ \\
\hline A2 & $\begin{array}{l}\text { No ensino fundamental } \\
\text { em turno diurno amostra } \\
\text { realizada com } 1.923 \\
\text { estudantes com idades } \\
\text { entre } 15 \text { a } 19 \text { anos. }\end{array}$ & $\begin{array}{l}\text { Nesse estudo, } 10 \% \text { dos alunos } \\
\text { pesquisados apresentaram sintomatologia } \\
\text { depressiva com maior predominância no } \\
\text { sexo feminino. }\end{array}$ \\
\hline A3 & $\begin{array}{l}\text { O estudo realizado } \\
\text { aleatoriamente com uma } \\
\text { amostra representativa } \\
\text { de } 1.265 \text { adolescentes de } \\
\text { idades de } 11 \text { a } 15 \text { anos } \\
\text { em zona urbana. }\end{array}$ & $\begin{array}{l}\text { Esses resultados retratam que os } \\
\text { adolescentes na fase inicial tiveram uma } \\
\text { taxa de prevalência de sintomas } \\
\text { depressivos de } 2,1 \% \text {. }\end{array}$ \\
\hline A4 & $\begin{array}{l}\text { Uma pesquisa realizada } \\
\text { com } 222 \text { adolescentes } \\
\text { em uma escola pública } \\
\text { entre idades entre } 14 \text { a } \\
19 \text { anos. }\end{array}$ & $\begin{array}{l}\text { Os sintomas foram descritos pelos } \\
\text { adolescentes nesse estudo com as } \\
\text { palavras e proporções de tristeza } 20 \% \text {, } \\
\text { solidão } 20 \% \text {, desânimo } 19 \% \text {, choro } 12 \% \text {, } \\
\text { doença } 12 \% \text {, ideias de morte } 8 \% \text {, falta de } \\
\text { apetite } 5 \% \text { anaústia } 4 \% \text {. }\end{array}$ \\
\hline A5 & $\begin{array}{l}\text { Estudo realizado em uma } \\
\text { escola de ensino médio } \\
\text { da rede pública com uma } \\
\text { amostra de } 275 \text { alunos na } \\
\text { faixa etária de } 14 \text { a } 17 \\
\text { anos. }\end{array}$ & $\begin{array}{l}\text { Essa pesquisa obteve o resultado de } 4 \% \\
\text { da amostra geral apresentaram } \\
\text { sintomatologia depressiva caracterizando } \\
\text { a depressão ser: sinônimo de tristeza, } \\
\text { desilusão amorosa (dor), solidão e } \\
\text { ideação suicida. Resultados coletados a } \\
\text { partir da análise por desenhos realizados } \\
\text { por estudantes }\end{array}$ \\
\hline A6 & $\begin{array}{l}\text { Amostra realizada em } \\
\text { escolas da rede pública e } \\
\text { privada com } 505 \\
\text { adolescentes com idades } \\
\text { entre } 14 \text { e } 16 \text { anos. }\end{array}$ & $\begin{array}{l}\text { O estudo apontou uma prevalência de } 5 \% \\
\text { apresentaram a sintomatologia depressiva } \\
\text { na população geral, ambos os sexos, os } \\
\text { sintomas identificados foram: tristeza, } \\
\text { pensamento suicida e de morte, choro e } \\
\text { insônia houve predominância no sexo } \\
\text { feminino. }\end{array}$ \\
\hline A7 & $\begin{array}{l}\text { Amostra realizada com } \\
135 \text { adolescentes de } 10 \text { a } \\
17 \text { anos, moradores de } \\
\text { uma região de alta } \\
\text { vulnerabilidade social. }\end{array}$ & $\begin{array}{l}\text { Foram apresentados perda e ganho de } \\
\text { peso, baixa autoestima, humor deprimido } \\
\text { e irritável, e fadiga foram questões de } \\
\text { maior explicação dos sintomas } \\
\text { depressivos onde tiveram prevalência no } \\
\text { sexo masculino. }\end{array}$ \\
\hline
\end{tabular}


Os principais sintomas identificados foram à falta de rede de apoio como distanciamento da família e adversidades familiares, bem como baixa autoestima e insatisfação com a vida atual. O sintoma mais raro foi associação com bebidas alcoólicas, o que pode ser consequência de uma reunião de fatores.

Com relação ao gênero, as mulheres desenvolveram depressão mais que homens e a correlação com as tentativas suicidas também foi maior para as mulheres.

Quadro 03- fatores relacionados às causas da sintomatologia, que levam a depressão na adolescência

\begin{tabular}{|c|c|}
\hline ARTIGO & PRINCIPAIS SINTOMAS \\
\hline $\mathrm{A} 2$ & $\begin{array}{l}\text { Nesse estudo a população estudada associou os sintomas } \\
\text { depressivos vivenciados, devidos aos fatores como: familiares onde } \\
\text { a estrutura familiar é menos preservada composta por padrasto e } \\
\text { madrasta; relacionamento regular/ruim entre pais e irmãos; } \\
\text { ausência de supervisão familiar; fraco apoio emocional e baixa } \\
\text { interação positiva. A ocorrência de eventos estressantes na família, } \\
\text { problemas financeiros uso de álcool e drogas, separação dos pais, } \\
\text { revelam que o adolescente apresenta } 73 \% \text { chances de } \\
\text { apresentarem sintomas depressivos comparados a aqueles que } \\
\text { nunca passaram por essa experiência, severa violência cometida } \\
\text { pelo pai e mãe tem } 6,5 \% \text { mais chances. E nas questões individuais } \\
\text { os insatisfeitos com a vida apresentam } 3,2 \% \text { mais chances. }\end{array}$ \\
\hline A3 & $\begin{array}{l}\text { Diante dessa analise a sintomatologia esteve associada às } \\
\text { seguintes causas: baixa condição socioeconômica, ausência } \\
\text { escolar no ano anterior, baixo rendimento acadêmico, não praticar } \\
\text { religião, ter tomado porre ou ter ingerido bebida alcoólica no último } \\
\text { mês. }\end{array}$ \\
\hline A4 & $\begin{array}{l}\text { Observou-se nesses resultados que esses sintomas da depressão } \\
\text { na adolescência aos fatores psicossociais, e fatores sociais; } \\
\text { adversidades ao ambiente familiar. Baixo autoestima } 37 \% \text {, } \\
\text { problemas familiares } 26 \% \text {, exclusão social } 19 \% \text {, perda } 8 \% \text {, } \\
\text { estresse } 5 \% \text {, e falta de diálogo } 5 \% \text {. }\end{array}$ \\
\hline A5 & $\begin{array}{l}\text { De acordo com os desenhos realizados pelos alunos foram } \\
\text { classificados a causa da depressão: relacionados aos aspectos } \\
\text { psicoafetivos, quando estava relacionado ao fator psicossocial e as } \\
\text { idéias mórbidas. }\end{array}$ \\
\hline A7 & $\begin{array}{l}\text { Adolescentes em situações de vulnerabilidades sociais estão } \\
\text { expostos a desenvolver sintomas depressivos por ficar por } \\
\text { períodos de } 2 \text { a } 4 \text { horas seguidas em frente a televisão, } \\
\text { computador ou videogame fazendo uso de jogos com conteúdos } \\
\text { violentos pelos meninos. }\end{array}$ \\
\hline
\end{tabular}

Identificou-se que a maioria dos estudos selecionados na presente pesquisa foi realizada no Nordeste do Brasil, podemos inferir que o interesse maior sobre a temática nessa região do país pode estar relacionado com os frequentes ataques preconceituosos e demais formas de bullying aos brasileiros nordestinos, que 
consequentemente, pode influenciar no desencadeamento de transtornos depressivos.

Contudo, os resultados do presente estudo permitiram evidenciar que a adolescência é uma fase de intensas mudanças fisiológicas e psicossociais que afetam o jovem na maturação entre a infância e a vida adulta (CAVALCANTE et al., 2007).

Sobre os aspectos clínicos da depressão em crianças adolescentes, um levantamento feito através de um estudo manual sintetizou que as manifestações da depressão em adolescentes não se apresentam apenas tristes, pois, apresentam humor irritável e instável podendo ocorrer crises de explosão e raiva em seu comportamento (BAHLS, 2002).

Entretanto para Moreira et al (2016), os sintomas depressivos podem levar à disforia como humor irritável, angústia, ansiedade, inquietação e agressividades e podem ser entendidos como dificuldades de lidar com os sentimentos, baixo auto estima, desamparo, alterações do sono com frequente hipersônica, o abuso de álcool e outras drogas solidão e violência física.

Para Santos et al, (2013), os principais sintomas apresentados pelos adolescentes foram a tristeza, a labilidade emocional, choro fácil, presente em 56 adolescentes (61,5\%), seguidos dos distúrbios do sono com insônia (45,1\%) 41 dos casos.

No que se refere à prevalência de sintomas depressivos, Jatobá e Bastos (2007) identificaram em 59,9\% dos 243 estudantes pesquisados apresentavam sintomatologia compatível e destacaram que essa prevalência foi maior que os estudos comparados.

Quanto ao gênero, as manifestações sintomatológicas depressivas explanadas têm mais frequência no sexo feminino de acordo com as pesquisas realizadas (RIBEIRO; COUTINHO; NASCIMENTO, 2010; SANTOS, et. al, 2013) sendo que as meninas apresentam maior risco de desenvolver o transtorno após a puberdade (MARTANI, 2007).

Em relação à ideação suicida um estudo analítico do tipo transversal no sul do Brasil com uma amostra composta por 1.118 que tinham em média idades de 14 a 16 anos, a prevalência para o pensamento suicida foi de $13.8 \%$ e $10,5 \%$ para o planejamento de suicídio e 5,55 para as tentativas de suicídio, onde o sexo masculino tinha a maior incidência (70\%) pertenciam a classe ao nível econômico alto (GONÇALVES, 2016).

Uma revisão de literatura apontou que a ideação suicida tem uma alta prevalência e está relacionada a uma gama de fatores que estão associados a desesperança, solidão, ansiedade, agressão por parte dos pais. A presença dos sintomas depressivos este estudo destacou um importante fator para a ideação suicida (MOREIRA et al., 2015).

Um estudo realizado na cidade de Porto Alegre com 526 adolescentes demonstrou que 188 adolescentes com ideação suicida, todos conheciam alguém que tenha tentado o suicídio e que o sexo feminino comparado ao masculino apresentou 2,1 vezes maior, onde confirma que $28 \%$ apresentaram a depressão onde é e um dos fatores de risco que mais está associado com esse tipo de comportamento (BORGES et al., 2016).

Uma pesquisa demonstrou que a violência doméstica independente do tipo de agressor (pai, mãe avós, irmãos etc.) é um fator de risco potencial para o 
desenvolvimento de problemas mentais na adolescência como a depressão (HILDEBRAND et al., 2015).

Entretanto, a sintomatologia da depressão está ancorada a uma diversidade de conceitos que estão associados a dimensões psicológicas, psicossocial, afetivo, comportamental e físico-orgânica. Nesse sentido, na esfera familiar, a violência psicológica é o fator que mais predispõe adolescentes ao desenvolvimento de transtornos de origem psiquiátrica (AVANCl et al., 2007).

\section{CONCLUSÃO}

Os estudos confirmam uma elevada prevalência no quadro depressivo vivenciados por essa população, onde se manifestam com maior frequência no sexo feminino. Nesse sentido, tristeza, humor deprimido, solidão, desânimo e sentimento de culpa, falta de apoio e desarranjos familiares possuem forte ligação com o comportamento suicida e podem levar ao suicídio.

Diante do exposto, sugere-se que no ambiente escolar sejam articuladas estratégias de saúde junto aos os educadores, que façam abordagem desse tema explicitando sobre os sintomas apresentados e quais as principais consequências. Dessa forma, sugere-se a realização de ações educativas que promovam e contribuam com a melhora da autoestima e com o incentivo à construção de estratégias de enfrentamento que podem contribuir para um melhor desenvolvimento dos adolescentes e amenizar os sintomas depressivos apresentados durante essa fase.

Concluiu-se também que deverão ser realizados novos estudos sobre a temática para que se possa implementar medidas de prevenção aos transtornos depressivos e do suicídio com vistas a identificar precocemente e intervir para uma melhor qualidade de vida dos adolescentes.

\section{REFERÊNCIAS}

ARAGAO, T. A.; COUTINHO, M. da P. de L.; ARAUJO, L. F. de; CASTANHA, A. R.. Uma perspectiva psicossocial da sintomatologia depressiva na adolescência. Ciênc. saúde coletiva [online]. 2009, vol.14, n.2, pp.395-405. ISSN 1413-8123. http://dx.doi.org/10.1590/S1413-81232009000200009.

AVANCI, J. Q.; ASSIS, S. G.; OLIVEIRA, R. V. C.. Sintomas depressivos na adolescência: estudo sobre fatores psicossociais em amostra de escolares de um município do Rio de Janeiro, Brasil. Cad. Saúde Pública [online]. 2008, vol.24, n.10, pp.2334-2346. ISSN 0102-311X. http://dx.doi.org/10.1590/S0102$311 \times 2008001000014$.

BARBOSA, D. G.; ANDRADE, R. D.; TEIXEIRA, C. S.; GOMES NETO, M.; FELDEN, É. P. G.. Sintomas depressivos em adolescentes em situação de vulnerabilidade social. Cad. saúde colet. [online]. 2016, vol.24, n.2, pp.221-227. Epub June 23, 2016. ISSN 1414-462X. http://dx.doi.org/10.1590/1414-462X201600020195.

BIAZUS, C. B.; RAMIRES, V. R. R.. Depressão na adolescência: uma problemática dos vínculos. Psicol. estud., Maringá , v. 17, n. 1, p. 83-91, Mar. 2012. Available from <http://www.scielo.br/scielo.php? script=sci_arttext\&pid=S1413- 
73722012000100010\&Ing=en\&nrm=iso >. access on 07 Mar 2018. http://dx.doi.org/10.1590/S1413-73722012000100010

BAHLS, S. Aspectos clínicos da depressão em crianças e adolescentes: clinical features. J. Pediatr. (Rio J.) [online]. 2002, vol.78, n.5, pp.359-366. ISSN 00217557. http://dx.doi.org/10.1590/S0021-75572002000500004.

BRAGA, D.. A adolescência e seu significado evolutivo. 2013. Porto Alegre: Artmed.

BOtTINO, S. M. B.; BOTTINO, C. M. C.; REGINA, C. G.; CORREIA, A. V. L.; RIBEIRO, W. S.. Cyberbullying and adolescent mental health: systematic review. Cad. Saúde Pública, Rio de Janeiro, v. 31, n. 3, p. 463-475, Mar. 2015. Available from <http://www.scielo.br/scielo.php?script=sci_arttext\&pid=

S0102-311X2015000300463\&lng=en\&nrm=iso>. access on 07 May 2018. http://dx.doi.org/10.1590/0102-311x00036114.

BORGES, J.C. Psicoterapias na adolescência e infância. Porto Alegre: Artmed. 2016.

COUTINHO, A. C. Observação do comportamento e ideação suicida em adolescentes. São Paulo: Atlas. 2016.

DAMIÃO, N. F.; COUTINHO, M. da P. de L.; ZULMIRA, C. G. C.; RIBEIRO, K. C. S.. Representações sociais da depressão no ensino médio : um estudo sobre duas capitais. Psicologia \& Sociedade, João Pessoa Abr 2011,vol.23,no.1, p.114-124.

DELL' ÁGLIO, J.C.; TAVARES DA SILVA, A. S., GUTHS, P. B.. Terapia cognitiva no tratamento da depressão. Psicoterapias. Porto Alegre: Artmed. 2013.

GOMES H. Transtornos do Humor. Porto Alegre: Artmed. 2013

GONCALVES, L. R. C.; GONCALVES, E.; OLIVEIRA JUNIOR, L. B. de. Determinantes espaciais e socioeconômicos do suicídio no Brasil: uma abordagem regional. Nova econ., Belo Horizonte , v. 21, n. 2, p. 281-316, Aug. 2011. Available from <http://www.scielo.br/scielo.php?script=sci_Arttext \&pid=S010363512011000200005\&lng=en\&nrm=iso>. access on 07 May 2018. http://dx.doi.org/10.1590/S0103-63512011000200005.

HILDEBRAND, J.C.. Well-Being and social capital. Social Research, v.81: p.455496, 2015.

KOLVALSKI, A. An economic theory of suicide. The journal of political economy. v.82, n.1, p.83-98, 2015.

JATOBA, J. D'Arc V. N.; BASTOS, O.. Depressão e ansiedade em adolescentes de escolas públicas e privadas. J. bras. psiquiatr., Rio de Janeiro, v. 56, n. 3, p. 171179 , 2007 Available from <http://www.scielo.br/scielo.php?script=sci_arttext\&pid=S0047-20852007000 
300003\&Ing=en\&nrm=iso >. access on $04 \quad$ Apr. 2018. http://dx.doi.org/10.1590/S0047-20852007000300003.

MARQUES et al HELLIWELL, J.H. Does suicide pose a puzzle? Indicators. 2014

MARTANI S. Uma viagem pela puberdade e adolescência. São Paulo: Aldeia Cultural; 2007.

MIRANDA. Religion, culture, economic and sociological correlates of suicide rates: a cross-national analysis. Applied Economics Letters, 3: 779-782, 2013.

MIRANDA VPN, CONTIMA, BASTOS RR, LAUS MF, ALMEIDA SS, FERREIRA MEC. Imagem corporal de adolescentes de cidades rurais. Ciência \& Saúde Coletiva, 19(6):1791-1801, 2014.

MONTEIRO, Fabiana Ribeiro; COUTINHO, Maria da Penha de Lima; ARAUJO, Ludgleydson Fernandes de. Sintomatologia depressiva em ddolescentes do ensino médio: um estudo das representações sociais. Psicol. cienc. prof., Brasília , v. 27, n. 2, p. 224-235, jun. 2007 . Disponível em <http://pepsic.bvsalud.org/scielo.php?script=sci_arttext\&pid=S141498932007000200005\&lng=pt\&nrm=iso>. acessos em 04 abr. 2018.

MOREIRA et al. Suicide and social change in China. Culture, Medicine and Psychiatry. 23: 1 (2015) 25-50. DOI: 10.1023/A:1005462530658

World Health Organization. Preventing suicide: A resource for media professionals. Geneva: World Health Organization, 2016. (Em Inglês)

RIBEIRO, K. C. S,; COUTINHO, M. da P. de L; NASCIMENTO, E. da S.. Representação social da depressão em uma Instituição de Ensino da Rede Pública. Psicol. cienc. prof., Brasília , v. 30, n. 3, p. 448-463, set. 2010 . Disponível em $<$ http://pepsic.bvsalud.org/scielo.php?script=sci_arttext\&pid $=$ S141498932010000300002\&lng=pt\&nrm=iso >. acesso em 04 abr. 2018.

SANTOS PINDYCK, R. Volatility in Natural Gas and Oil Markets. The Journal of Energy and Development 30(1):1-19, 2016

SOUZA, L. D. de M. S.; SILVA, R. S.; GODOY, R. V.; CRUZEIRO, A. L. S.; FARIA, A. D.; PINHEIRO, R. T.; HORTA, B. L.; SILVA, R. A. da. Sintomatologia depressiva em adolescentes iniciais: estudo de base populacional. J. bras. psiquiatr., Rio de Janeiro , v. 57, n. 4, p. 261-266, 2008 . Available from <http://www.scielo.br/scielo.php?script=sci_arttext\&pid $=S 0047-2085200 \quad 80004$ 00006\&lng=en\&nrm=iso $>$. access on $04 \quad$ Apr. 2018. http://dx.doi.org/10.1590/S0047-20852008000400006. 